\title{
Compact Multi-Anvil Wedge-Type High Pressure Apparatus
}

\author{
E. C. Lloyd, U. O. Hutton, and D. P. Johnson
}

(May 8, 1959)

\begin{abstract}
Apparatus for generating high pressures by application of force by an anvil against each face of a solid polyhedron is described. The equipment constructed utilizes a tetrahedron of pyrophyllite and four tungsten carbide anvils as described earlier by H. T. Hall. External force is applied to only one of the anvils, and wedge reaction forces act on the other anvils, permitting the equipment to be used in a conventional hydraulic press. Examples of results of use of the equipment are given. Success of the design has been shown by the repeated generation of pressures in excess of 100,000 atmospheres indicating that large existing presses might be used to generate pressures of this order in volumes of several cubic inches. For higher pressures a two-stage multi-anvil apparatus is proposed in which the second-stage anvils are embedded in a large pyrophyllite tetrahedron to obtain necessary supporting forces.
\end{abstract}

\section{Introduction}

A multiple-anvil apparatus for generating high pressures by application of force by an anvil against each face of a solid polyhedron is described. Such an arrangement was described by Hall ${ }^{1}$ for pressures up to 100,000 atmospheres using four independent hydraulic rams to apply force to the faces of a tetrahedron of pyrophyllite. The present equipment employs an assembly in which external force is applied only to one of the four anvils, and wedge reaction forces act on the remaining three anvils. This results in a compact equipment that can be used in a press of conventional design, with the further advantages of easy manipulation and rapid assembly and disassembly. Although apparatus of the type described here lends itself, in special applications, for use with solids having a larger number of faces, the regular tetrahedron appears to be the most generally desirable shape, and is the ouly one used with the apparatus so far.

This configuration has the attractive possibility of permitting the use of existing presses of very large capacity to obtain high pressures in volumes of several cubic inches. The availability of high pressure in volumes of this size would present the possibility of practical two-stage devices to obtain still higher pressures.

\section{Constructional Features and Operation}

Figure 1 shows the wedge-type tetrahedral assembly in place in a small hand-operated press used for applying ram forces up to 50 tons. Figures 2 and 3 illustrate the general construction, and the drawings of figures 4 and 5 show some of the details of the equipment. The initial apparatus, illustrated here,

\footnotetext{
${ }^{1}$ H. Tracy Hall, Rev. Sci. Inst. 29, No, 4, 267 (1958).
}

utilizes anvils having triangular faces $1 / 2$-inch on an edge (face area about $\frac{1}{9} \mathrm{in}^{2}{ }^{2}$ ) to accommodate tetrahedrons of pressure-transmitting material of $9 / 16$-inch edge-length. The half-angle of the inner surface of the conical ring is $18 \frac{1}{2}$ degrees.

In operation, the clamping force of a hydraulic press applied to the upper anvil and the bottom of the containing ring causes the three lower anvils to slide downward along the inner conical surface of the containing ring, and the resulting wedging forces result in substantially equal motion of all four anvils toward the center of the pyrophyllite tetrahedron.

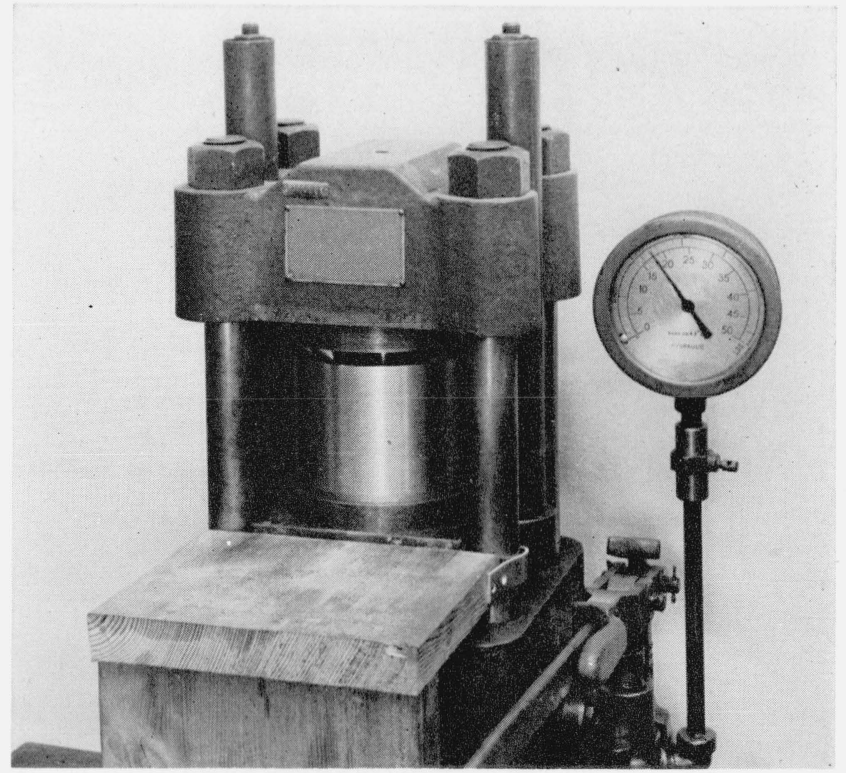

Figure 1. Wedge-type tetrahedral apparatus in place in a 50-ton hand-operated press for use in pressure range up to 60,000 atmospheres. 
Measurements made during initial tests of the equipment at 100 tons load (calculated pressure near 125,000 atm) showed deformation of the conical ring into a three-lobed shape that fitted inside a circle about 0.001-inch larger in diameter than the unloaded ring. Based on this deformation, stresses in the ring appear to be consistent with design stresses, which were selected to be

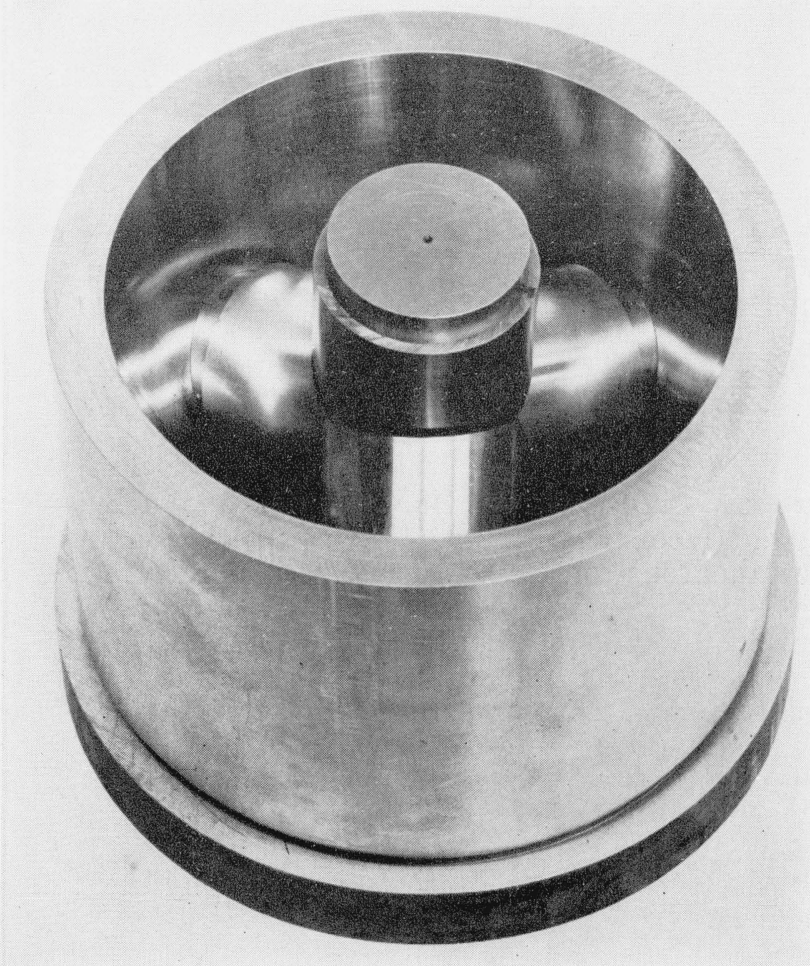

FIGURE 2. View of wedge-type apparatus with upper bolster removed showing arrangement of anvils in conical ring.

The butt-ends of the three lower anvils are shaped so that in their lowest position they exactly fit the ring.

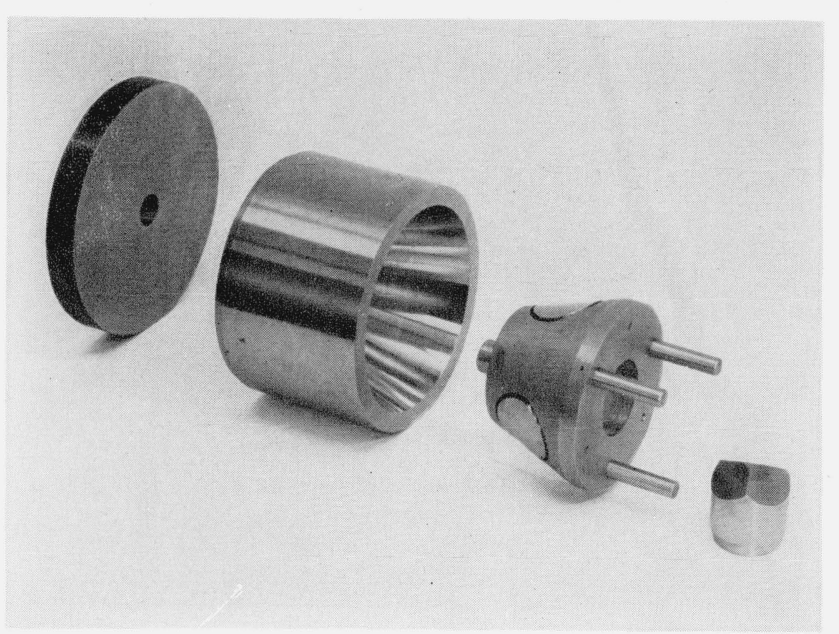

Figure 3. Disassembled view of apparatus, with lower anvils installed in conically shaped guide block used for machining the butt-faces. conservative with respect to the strength of the SAE 4340 steel. No evidence of permanent deformation of the ring or anvils has been found after more than 10 cycles to a ram load of 100 tons.

The machining of the butt-faces of the three lower anvil assemblies (to conform to the shape of the ring in the final position of the anvils) was done with the use of the guide block shown (fig. 3). This block was originally also used during application of load to guide the anvils. This was found to be unnecessary, however, because of the selflocating effect of the faces of the wedges and their low frictional reaction in the cone, and the use of the guide block for this purpose was discontinued in order to simplify electrical insulation of the anvils.

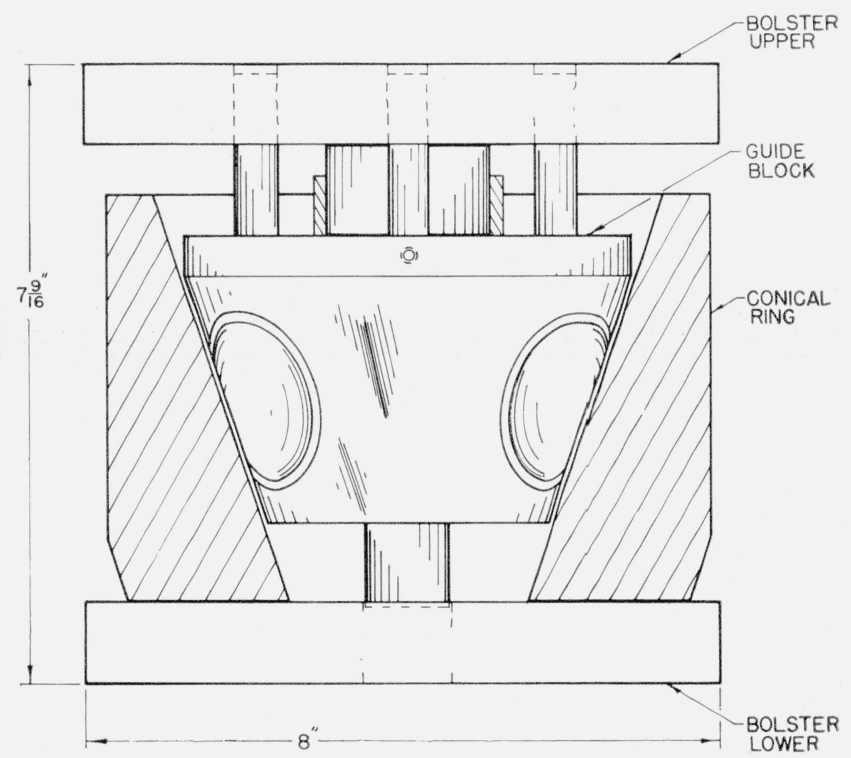

Figure 4. Sectional drawing of wedge-type apparatus. showing guide block inside conical ring.

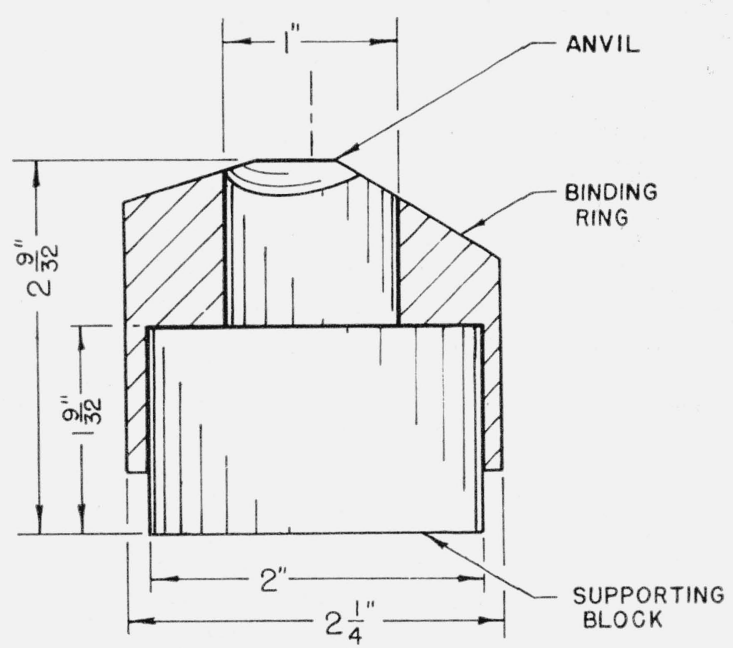

Figure 5. One of the four three-piece anvil assemblies, showing the tungsten carbide anvil supported by a steel binding ring and supporting block. 
The material used for the anvil assemblies and conical ring was SAE 4340 steel heat treated to Rockwell $40 / 41 \mathrm{C}$, except the anvils which were of Allegheny Ludlum grade CA-4 tungsten carbide. The upper and lower bolster plates and the guide block were cold rolled steel.

The pressure-transmitting material used has been pyrophyllite (hydrous aluminum silicate, $4 \quad \mathrm{SiO}_{2}$. $3 \mathrm{Al}_{2} \mathrm{O}_{3} \cdot \mathrm{H}_{2} \mathrm{O}$ ) and, as in Hall's equipment, this pressure medium extrudes between rouged surfaces of the anvils to form a compressible gasket. The specimen to be studied is contained in a cavity inside the tetrahedron, frequently a hole drilled between centers of opposite edges. The specimen size has been varied from 5 to 50 percent of the tetrahedral volume, but if the specimen has been more than 10 percent of this volume difficulty in preventing extrusion of the specimen through the gasket has frequently been encountered. This difficulty is decreased by more precise fitting of the tetrahedron.

With pyrophyllite tetrahedrons of $9 / 16$-inch initial edge-length, measurements made in initial trials of the equipment showed the total vertical motion of the upper anvil to be about 0.030 -inch as the load was increased from 5 tons to 100 tons. The stroke for the initial 5-ton load varied depending on the amount of "slack" in the system. The time required to reach a load of 100 tons could be selected over a wide range, and any intermediate load could be held for periods up to several hours without difficulty.

Using $9 / 16$-inch tetrahedrons the extrusion of pyrophyllite forms a gasket between the parallel anvil surfaces having a width of about $3 / 32$-inch, and a thickness of about 0.006-inch, as shown in figure 6 . In order to obtain an equal gasket thickness in all gasket areas the three lower anvils are initially spaced with plastic buttons near the tetrahedral die area, of thickness to provide symmetrical positioning of the four anvils around the over-sized tetrahedron inserted in the cavity. Spacers of indium, block tin, or Teflon (when a nonconductor is desired), have been used with equal success, and the gasket is ordinarily extruded in quite a symmetrical configuration on all edges.

One of the difficulties to be expected in designing this equipment lies, of course, in overcoming the effects of excessive friction between the sliding anvils and the conical ring. A number of materials have a low coefficient of friction under heavy loading and appeared to be suitable for use as lubricants between these surfaces. Sheet Teflon 0.003-inch thick was first tried, and found to be so satisfactory that no other material has been used. In addition to serving as a lubricant the Teflon provides electrical insulation between the anvils and the ring. Tests on 0.003-inch sheet Teflon using the method ${ }^{2}$ of measuring the torque required to turn a smooth right circular cylinder loaded longitudinally with the sample to be tested as a lubricating film at each end of the cylinder gave the values of friction listed in table 1.

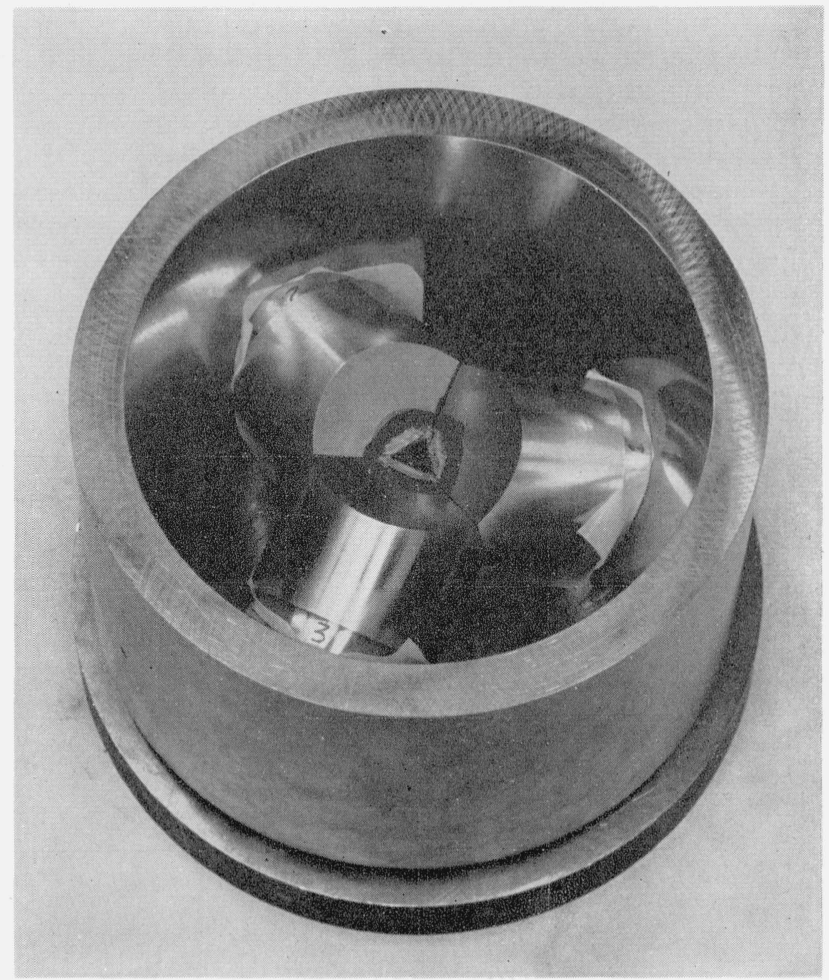

FIGURE 6. Wedge-type apparatus with top anvil removed, after application of pressure, showing pyrophyllite tetrahedron in place.

The gasket is the light area adjacent to tetrahedron, and the surrounding darker area is rouge coating. Sheets of Teflon to act as lubricant are injplace between the bases of the anvil assemblies and the conical ring.

TABLE 1. Coefficient of dry friction between sheet Teflon and smooth steel

\begin{tabular}{c|r|r}
\hline $\begin{array}{c}\text { Average } \\
\text { pressure }\end{array}$ & $\begin{array}{c}\text { Starting } \\
\text { friction }\end{array}$ & $\begin{array}{c}\text { Sliding } \\
\text { friction }\end{array}$ \\
$p s i$ & & \\
33,000 & 0.011 & 0.007 \\
66,000 & .012 & .008 \\
83,000 & .014 & .010 \\
& & \\
\hline
\end{tabular}

These values of coefficient of friction correspond to a sliding angle of about $1 / 2$ to 1 degree; accordingly, the half angle of the conical surface of the ring was made 18.5 degrees, or one degree smaller than the angle complementary to the angle between faces of the tetrahedron (70.528 degrees). This results in the forces exerted on the lower three anvils being within $1 / 2$-degree of the axes of the anvils.

The adequacy of the Teflon lubricant has been well demonstrated in more than 30 force applications, of which about 10 were in the neighborhood of 100 tons, without any evidence of metal-to-metal contact. In one test the same Teflon pieces were used in three successive force applications at 100 tons without failure.

Measurements of the dimensions and density of pyrophyllite tetrahedrons after being subjected to various pressures showed that this material increases

\footnotetext{
2 P. W. Bridgman, Phys. Review 48, 825 (1935).
} 
nearly irreversibly in density with pressure, according to the curve of figure 7 . The material exhibited relatively small elasticity in compression, so that only small increases in volume occurred after release of pressure.

Specimens to be subjected to pressure are inserted in a hole (up to $1 / 8$ in. diameter) drilled from edge to edge of the tetrahedron, as may be seen in figure 8 . In order to study the extent to which the pressure over such a specimen departs from true hydrostatic pressure, cylinders of indium were used, and the resulting deformations noted. Such a specimen is

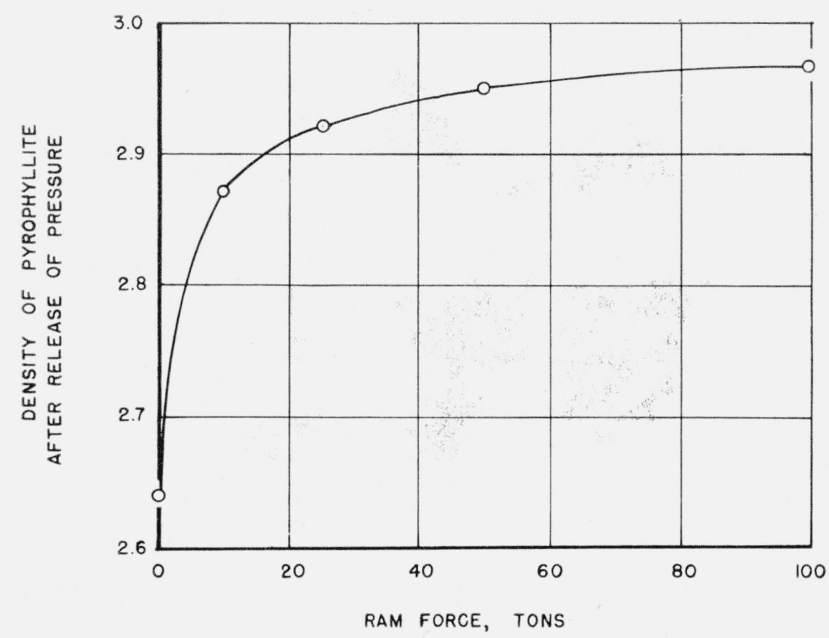

FIguRe 7. Permanent increase in density of pyrophyllite due to application of pressure. shown in figure 8. In specimens which were not extruded through the gasket there is a characteristic flattening of the ends parallel to the adjacent edges of the tetrahedron. The shear yield strength of indium under high pressures, comparable to those encountered here, is reported by Bridgman to be about the same as at ambient pressure, about 1,000 psi. This suggests that the departure from uniform pressure over the surface of the indium specimen is only of the order of a few hundred atmospheres at most. A specimen material which is embedded, or sheathed, in an indium cylinder would thus be subjected to uniform pressure within pressure variations of this magnitude. Tests with still softer materials are planned to determine whether they can be contained by the pyrophyllite, and thus obtain a still better approximation to true hydrostatic pressure.

Figures 9 and 10 are examples of pressure-resistance relationships obtained on specimens of bismuth and antimony, respectively. In each case the volume of the specimen was approximately 4 percent of the volume of the tetrahedron. In the measurements of resistance of these specimens under pressure a fourlead circuit was used, in which two of the anvils served as current leads and the other two as potential leads. Connection to the specimen was by use of silver foil strips as described by Hall, and as illustrated by one of the tetrahedrons of figure 8. In investigations requiring heating of the specimen this arrangement of electrical connections is available, of course, for conducting heating current and for tem-

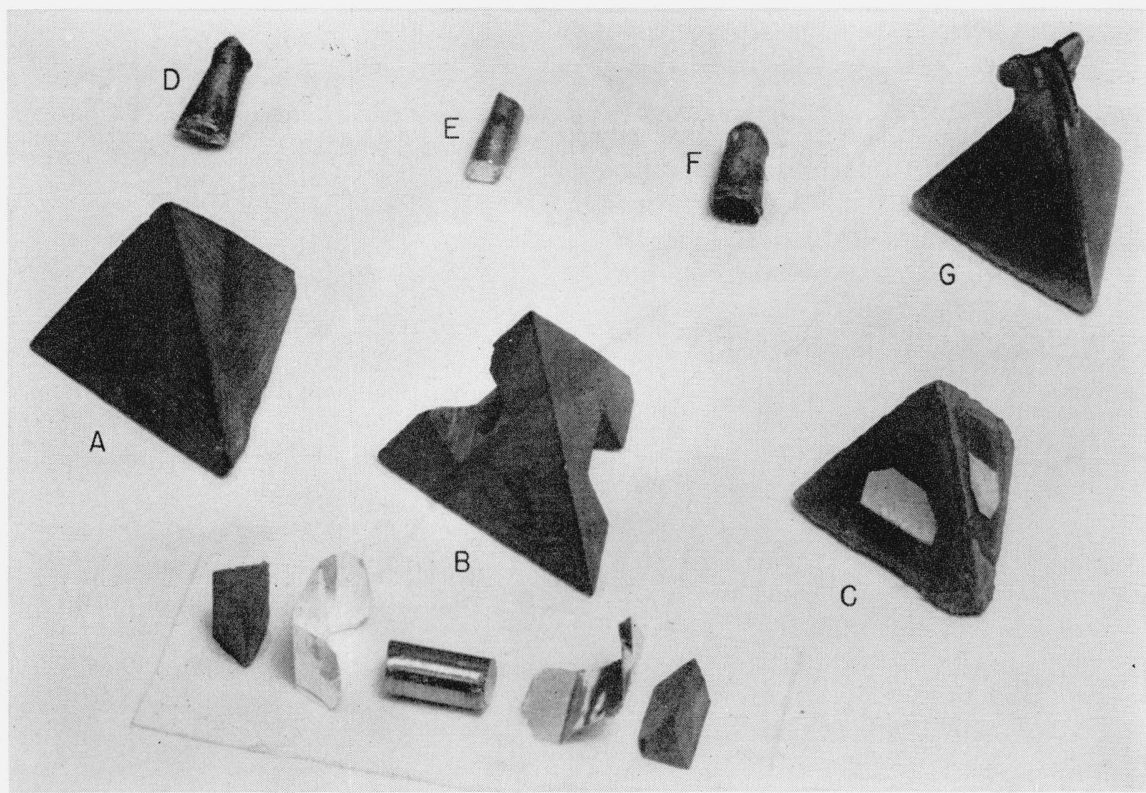

Figure 8. A. Pyrophyllite tetrahedron as cut. B. Pyrophyllite tetrahedron with drilled hole to receive specimen shown, with silver foil contacts and prisms of phrophyllite to fill notches at ends of hole. C. Pyrophyllite tetrahedron, assembled as in B, after removal from high pressure equipment. D, E, F. Specimens of indium, bismuth, and antimony, respectively, after release of pressure. Specimens were originally of cylindrical shape. G. Pyrophyllite tetrahedron after removal from high pressure equipment, showing example of extrusion of specimen of bismuth. 
perature measurements, and additional electrical connections would be available in a configuration using other shapes having a larger number of faces and anvils.

In figure 9 pressure is shown in terms of ram force for increasing and decreasing pressure. Superimposed is the pressure-resistance curve by Bridgman in which the pressure scale (abscissa) is linear; the first transition points on increasing pressure have been made to coincide. The abrupt fall in resistance of bismuth reported by Bridgman ${ }^{2}$ to occur at 25,000 atm is evident in figure 9. Bridgman's second transition at 27,000 atm (increasing resistance) is not sharply defined as to point of onset, but the maximum in resistance is definite. The displacement of the ascending and descending force versus resistance curves

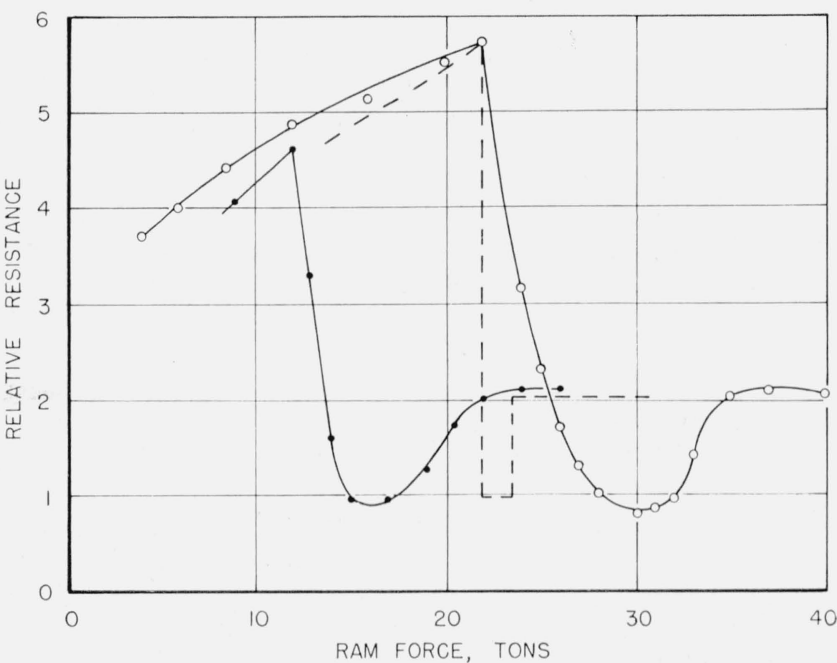

FIGURE 9. Ram force versus resistance of specimen of bismuth, 99.99 percent pure.

. Increasing force; $\boldsymbol{O}$, decreasing force. Bridgman's results, plotted to a linear pressure scale, are shown by the dashed line for comparison.

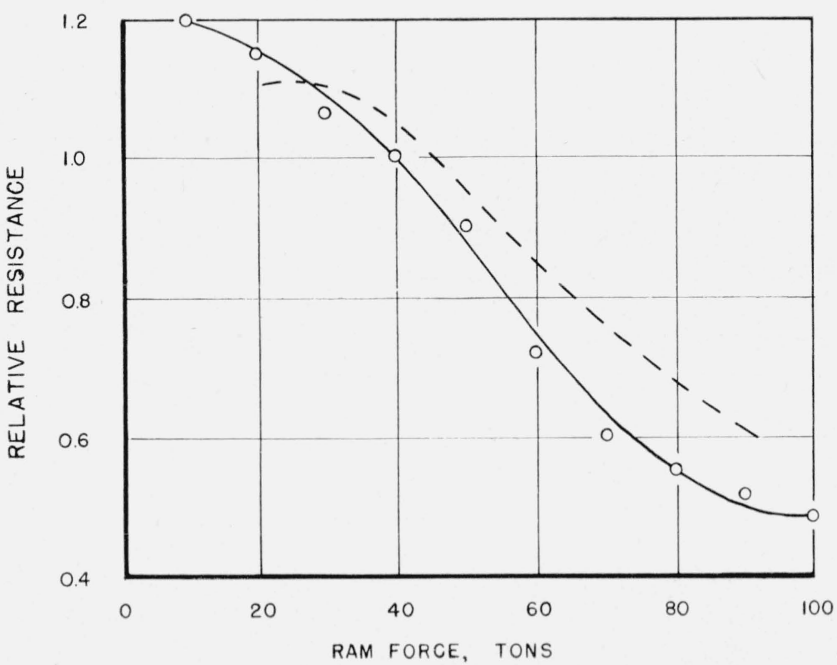

FIGURE 10. Ram force versus resistance of antimony, 99.99 percent pure, on increasing force.

Bridgman's results, plotted to a linear pressure scale, are shown by the dashed line for comparison. gives an indication of the hysteresis of this particular configuration of pyrophyllite and bismuth. The appreciable slope of our curve and the relatively wide force range over which the resistance transitions occur is probably due principally to two factors: the departure from uniform pressure over the surface of the bismuth due to internal friction in the pyrophyllite, and a departure from proportionality between ram load and specimen pressure during the constantpressure reductions in volume of bismuth at its two transition points (which amounts to $4 \frac{1}{2}$ percent and 3 percent for the first and second points, respectively.) This departure from proportionality evidently results from the additional gasket compression required to effect the reduction in bismuth volume at constant pressure. On decreasing pressure it will be noted that both transitions are displaced to ram forces coresponding to pressures considerably lower than Bridgman's values. This hysteresis is attributable partly to pressure "held" by the hysteresis effect of the pyrophyllite, which appears to increase significantly with the irreversible increase in density on application of pressure, and partly to friction between the anvils and the gasket.

Figure 10 shows results for antimony, in which the relative resistance changes continuously up to the highest pressure reached, probably over 100,000 atm. Although a volume discontinuity is reported by Bridgman ${ }^{2}$ at 85,000 atm, he found no corresponding specific resistance change at this pressure. The antimony used in the present case was probably of substantially higher purity than Bridgman's sample. Bridgman's result is plotted for comparison, on a linear pressure scale derived from the lower curve of figure 11 .

Estimates of the pressures obtained have initially been by measurement of the ram force and the areas of pyrophyllite under pressure. Two principal uncertainties are involved in this method: the reduction in effective ram force due to the force supported by the extruded gasket, and the effect of internal friction in the pyrophyllite. Measurements made on the gaskets formed by single applications of force from 25 tons to 100 tons to $9 / 16$-inch tetrahedrons gave a gasket lateral area, nearly independent of the force, of approximately 50 percent of the total area subjected to ram force. If the pressure in the gasket falls linearly from the edge of the tetrahedron to the free edge of the gasket, the net force acting on each face of the tetrahedron is approximately 75 percent of the total ram force. ${ }^{3}$ However, the loss in pressure, on increasing force, due both to gasket forces and to internal friction of pyrophyllite appears to be only about 10 percent, as indicated by values of ram force required to reach the first resistance transitions of bismuth and barium. The lower line plotted in figure 11 was drawn from data obtained on transitions of bismuth and barium, and the upper line is the calculated average pressure on the faces of the tetrahedron without correction made for gasket force.

\footnotetext{
${ }^{2}$ P. W. Bridgman, Phys. Review 48, 825 (1935).

${ }^{3}$ Actually, the variation in pressure in the gasket probably lies between a' linear and an exponential function, and the force supported by the gasket is
} appreciably less than 25 percent. 


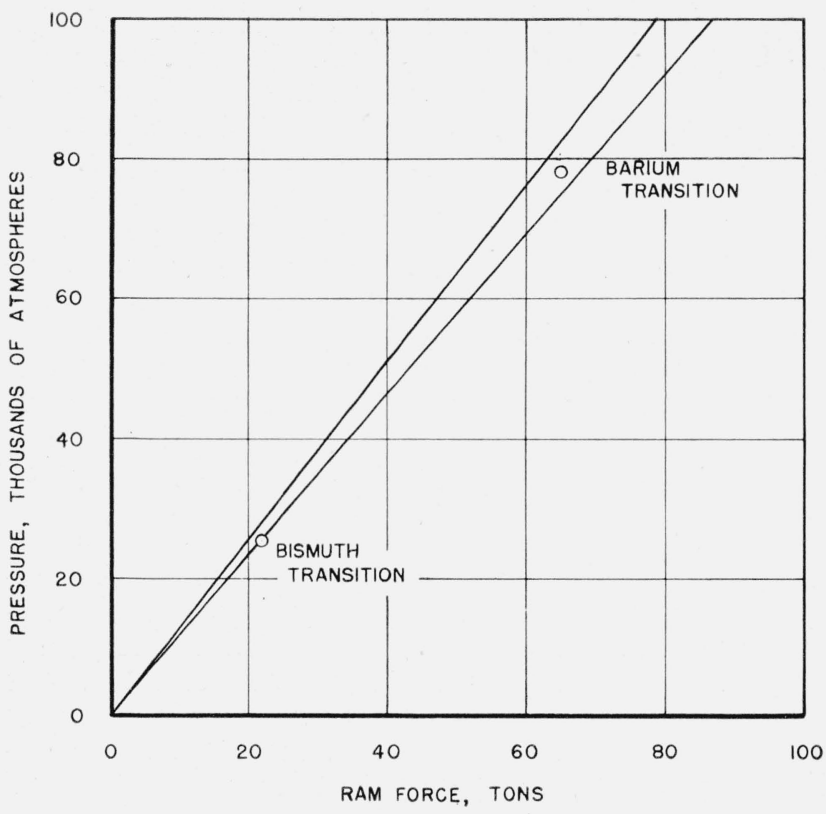

Figure 11. Calculated average pressure on face of $1 / 2$-inch tetrahedron (upper curve), and actual pressure on cylindrical specimen (lower curve) versus ram force.

The lower curve is based on the bismuth transition of figure 9 , with a point similarly obtained for barium shown for reference. The value of pressure for barium is due to Bridgman (78,000 atm).

The difference in ordinates of these two lines indicates the lag in pressure due to internal friction in the pyrophyllite and the loss of pressure due to force taken by the gasket.

\section{Future Work}

This high pressure equipment will be used, as well as stepped-piston equipment, to study properties of materials at high pressures, with the objective of establishing "fixed points" on the pressure scale, and of devising improved pressure measurement techniques.

Further investigations of pressure-transmitting media appear desirable to provide improved performance of the equipment in various pressure ranges. Combinations of tetrahedron material and specimen sheath material having more nearly optimum properties may substantially improve the uniformity of pressure applied to a specimen.

The use of a larger tetrahedron would improve the ratio of tetrahedron volume to specimen volume, should reduce the proportion of force taken by the gasket, and would evidently provide more uniform pressure in the central volume. New apparatus is under design to apply 600 tons to tetrahedrons of about 1-inch edge-length, to investigate the advantages of larger size.

The ultimate pressure attainable with the present equipment will be determined with the use of larger presses that will be available for further work. The absence of failure of any sort with repeated ram loads of 100 tons, the relatively low stresses in the conical ring, and the excellent performance of the teflon lubricant, give an indication that substantially higher ram loads may be used.

The adaptability of the equipment to conventional hydraulic presses makes feasible consideration of the use of very large ram forces, with a corresponding increase in size of the tetrahedron to perhaps $2 \frac{1}{2}$ or 3 inches. The availability of high-pressure volumes of this size may make a two-stage device practicable. Such a device is proposed, utilizing a large tetrahedron, in each face of which is embedded a second stage one-piece carbide anvil of such dimensions as to enclose a small tetrahedron at the center. The four second stage anvils would probably be tapered with the small ends faced to accommodate a tetrahedron and its gasket, as in the present equipment, and with the large ends in contact with the faces of the primary anvils. The secondary anvils would be subjected to the pressure of the large tetrahedron over their lateral surfaces to provide the necessary supporting forces. An alternative to the use of two sets of anvils is the machining of each anvil pair as one piece. It seems likely that adjustment of the parameters available, including relative dimensions of the large and small anvils, the elastic properties of the anvils and of the pressure transmitting media, and the relative volumes of large and small tetrahedrons, would provide a good chance of success of a two-stage device. Important in the proposed twostage device is the availability of four electrical connections into the highest pressure volume via the four anvil pairs.

We are indebted to Dr. H. T. Hall of Brigham Young University for information on the construction of his tetrahedral anvil equipment. Mr. H. J. Zoranski of the Mechanical Instruments Section prepared shop drawings of the equipment and made suggestions that were valuable in selecting the design finally adopted. The excellent machine work of the NBS Shops Division was an important factor in successful performance of the equipment.

Washington, D.C.

(Paper 63C1-6) 